\title{
A randomized placebo-controlled study: Phellodendron Bawei tablets combined with standard management can improve storage symptoms, sleep quality, and medication compliance in patients with benign prostatic hyperplasia compared to placebo with standard management
}

\author{
Yu-Chen Gong, Xiang Chen, Qing-Tian Song, Yu Gan, Bo Zhang, Bing-Sheng Li, Zhi Chen, Yao He \\ Department of Urology, Xiangya Hospital, Central South University, Changsha, China \\ Contributions: (I) Conception and design: YC Gong; (II) Administrative support: Y He; (III) Provision of study data or patients: Y He; (IV) Collection \\ and assembly of data: YC Gong; (V) Data analysis and interpretation: YC Gong; (VI) Manuscript writing: All authors; (VII) Final approval of \\ manuscript: All authors. \\ Correspondence to: Yao He, MD. Department of Urology, Xiangya Hospital, Central South University, Xiangya, Road 88, Changsha 410008, China; \\ Email: heyao1984@163.com.
}

\begin{abstract}
Background: Benign prostatic hyperplasia (BPH) is a common micturition disorder in middle-aged and elderly males, and it is one of the most common urology-related diseases worldwide. However, standard therapeutic drugs ( $\alpha 1$-receptor blockers $+5 \alpha$ reductase inhibitors) do not provide anti-inflammatory or antiinfective effects. The Phellodendron Bawei tablet is a proprietary Chinese medicine with anti-inflammatory and anti-infective effects. Here, we analyzed whether the combination of standard therapeutic drugs and Phellodendron Bawei tablets has more advantages than placebo with standard management in improving the lower urinary tract symptoms (LUTs), sleep quality, sexual function, and medication compliance in patients with BPH.
\end{abstract}

Methods: This study was a prospective, double-blind, single-center, 6-month clinical trial in patients with BPH. Male patients, 45-75 years old, a history of moderate-to-severe BPH/LUTs for more than 6 months, moderate-to-severe LUTs [International Prostate Symptom Score (IPSS) $\geq 8$ ], maximum urine flow rate (Qmax) of $<15 \mathrm{~mL} / \mathrm{s}$, and prostate volume (PV) of $>30 \mathrm{~mL}$. All patients were randomly divided into two cohorts at baseline. The standard management $(\mathrm{SM})$ group was treated with tamsulosin + finasteride + placebo, while the experimental group was treated with tamsulosin + finasteride + Phellodendron Bawei tablets. The clinical indicators were as follows: Age, body mass index (BMI), blood prostate-specific antigen (PSA), PV, Qmax, IPSS; IPSS voiding subscore (IPSS-V), IPSS storage subscore (IPSS-S), and IPSS quality of life (IPSS-QOL)], five-item version of the International Index of Erectile Function (IEFF-5) score, Athens Insomnia Scale (AIS) score, and the Medication Adherence Questionnaire (MAQ). And adverse drug reactions were observed. Student's $t$-test was used to analyze results.

Results: We randomly divided 120 patients into two groups, with 60 patients in each group, and a total of 105 patients completed the study. IPSS-S $(\mathrm{P}=0.027)$ and AIS scores $(\mathrm{P}<0.001)$ improved more significantly in the Phellodendron Bawei tablets + SM group, and the MAQ score in this group was lower $(\mathrm{P}=0.003)$.

Conclusions: Phellodendron Bawei tablets combined with $\alpha 1$-receptor blockers and $5 \alpha$-reductase inhibitors can improve lower urinary tract symptoms associated with urine storage, sleep quality, and medication compliance in patients with benign prostatic hyperplasia compared to placebo with standard management.

Trial Registration: Chinese Clinical Trial Registry ChiCTR2100046463.

Keywords: Phellodendron Bawei tablets; benign prostatic hyperplasia (BPH); lower urinary tract symptoms (LUTs) 
Submitted Jun 07, 2021. Accepted for publication Aug 16, 2021.

doi: $10.21037 /$ tau-21-588

View this article at: https://dx.doi.org/10.21037/tau-21-588

\section{Introduction}

Benign prostatic hyperplasia (BPH) is a common micturition disorder in middle-aged and older adult males, and it is one of the most common urology-related diseases worldwide. The prevalence of clinical $\mathrm{BPH}$ in males over 50 years is $50-75 \%$ (1). It has a negative impact on the quality of life in many areas, resulting in disordered sleep, sexual dysfunction, and reduced sexual satisfaction, along with lower urinary tract symptoms (LUTs) (1).

Recently, an increasing number of published articles have reported that $\mathrm{BPH}$ may be an immune-mediated disease, and persistent inflammation is the key factor for its development and progression (2). Guidelines strongly recommend the use of $\alpha 1$-receptor blockers combined with $5 \alpha$ reductase inhibitors for treating patients with $\mathrm{BPH}$ with moderate-to-severe LUTs and risk of progression $(3,4)$, but neither of these drugs provides anti-inflammatory or antiinfective effects. Non-steroidal anti-inflammatory drugs (NSAIDs), commonly used in clinical practice, can be highly toxic, with their side effects mainly being gastrointestinal inflammation, upper gastrointestinal ulcers, and even perforation and bleeding, and additional side effects being related to the kidneys, central nervous system, hematological system, respiratory system, skin, and liver (5). The use of NSAIDs is also a risk factor for BPH (6). Although antibiotics can effectively control recurrent urinary tract infections due to BPH, long-term use of antibiotics results in many side effects, such as the alteration of the intestinal flora and increased abundance of drug-resistant bacteria, with renal insufficiency also often arising in older adult patients (7). Therefore, identifying effective and low-toxicity anti-inflammatory drugs that can replace NSAIDs and antibiotics may be an important means to strengthening treatment compliance, reducing recurrence rate, and improving the quality of life for patients with BPH.

In recent years, more plant extracts and proprietary Chinese medicines have been used in the treatment of $\mathrm{BPH}$ and related LUTs to obtain a good therapeutic effect $(8,9)$. The Phellodendron Bawei tablet is a proprietary Chinese medicine, and its main components are Cortex Phellodendri, pine-soot ink, Fructus Gardeniae, Radix Glycyrrhiza, Flos Carthami, Fructus Piperis Longi,
Pulvis Billis Bovis, and Resina Liquidambaris,with anti-inflammatory and anti-infective effects. Its main component, Cortex Phellodendri, contains berberine, palmatine, phellodendrine, and obacunone, which may exert a synergistic anti-infectious effect by downregulating the messenger RNA (mRNA) expression of tumor necrosis factor- $\alpha$ (TNF- $\alpha$ ), interleukin-1 (IL-1), interleukin-6 (IL-6), and cyclooxygenase-2 (COX-2) (10). Other ingredients, such as Radix Glycyrrbizae and Flos Carthami, are used as components of Chinese medicines for insomnia $(11,12)$. And there are no reports of serious adverse events of Phellodendron Bawei tablet. However, thus far, there has been no report concerning the effects of Phellodendron Bawei tablets on the improvement of LUTs and sleep quality in patients with $\mathrm{BPH}$.

Therefore, the purpose of this prospective study was to analyze whether the combination of standard therapeutic drugs ( $\alpha 1$-receptor blockers $+5 \alpha$ reductase inhibitors) and Phellodendron Bawei tablets confers greater advantages than do placebo with standard management in improving LUTs, sleep quality, sexual function, and Medication Adherence Questionnaire (MAQ) score in patients with $\mathrm{BPH}$. We present the following article in accordance with the CONSORT reporting checklist (available at https:// dx.doi.org/10.21037/tau-21-588).

\section{Methods}

\section{Patients}

This study is a prospective, double-blind, single-center, two-parallel clinical trial in patients with BPH. This study followed the ethical principles of the Declaration of Helsinki (as revised in 2013) and the Guidelines for Good Clinical Practice. The protocol was approved by the Medical Ethics Committee of Xiangya Hospital of Central South University (approval No. 201703544). All subjects provided written, informed consent before registration. Patients aged 45-75 years diagnosed with $\mathrm{BPH}$ in the outpatient clinic of Xiangya Hospital from September 2019 to July 2020 were enrolled and randomly divided into control and experimental groups using a random number method and allocation ratio is $1: 1$. 
Inclusion criteria were as follows: $45-75$ years of age; a history of BPH/LUTs for more than 6 months, moderateto-severe LUTs [International Prostate Symptom Score (IPSS) $\geq 8$ ], maximum urine flow rate (Qmax) of $<15 \mathrm{~mL} / \mathrm{s}$, and prostate volume (PV) of $>30 \mathrm{~mL}$. Patients with prostate cancer, urethral stricture, and urinary tract infection, as well as those with previous urethral surgery, absolute indications for surgery, and drug allergies, were excluded.

\section{Treatment}

The standard management (SM) group was treated with tamsulosin $0.2 \mathrm{~g}$ once a day $(\mathrm{qd})+$ finasteride $5 \mathrm{mg} \mathrm{qd}+$ placebo [5 tablets, three times a day (tid)] for 6months, while the experimental group (Phellodendron Bawei tablets $+\mathrm{SM}$ ) was treated with tamsulosin $0.2 \mathrm{~g} \mathrm{qd}+$ finasteride $5 \mathrm{mg} \mathrm{qd}+$ Phellodendron Bawei tablets (5 tablets, tid) for 6 months. It was difficult to distinguish between the placebo and Phellodendron Bawei tablets in appearance. We have the assigned person determined the grouping sequence, the person did not participate in the inclusion of the cases, and the distribution table was strictly kept. We also confirmed blindness for investigators and patients throughout the treatment period. The observation period was 6 months, and the indicators were as follows: (I) Clinical indicators including body mass index (BMI), blood prostate-specific antigen (PSA), PV, and Qmax; (II) LUTs, including IPSS, IPSS voiding subscore (IPSS-V), IPSS storage subscore (IPSS-S), and IPSS quality of life (IPSS-QOL) score ; (III) sexual function score according to International Index of Erectile Function (IIEF-5) score (13) (5-7 points for severe sexual dysfunction, $8-11$ points for moderate sexual dysfunction, 12-21 points for mild sexual dysfunction, and 22-25 points for normal erectile function) (13); (IV) sleep status score according to the Athens Insomnia Scale (AIS) (14) (0-3 points for no sleep disorder, 4-6 points for suspected insomnia, and 7 points or more for insomnia) (14); (V) MAQ (a total score of 8 points: 0 points for low compliance, 1-2 points for medium compliance, and 3 points for low compliance) (15). (VI) Observe for adverse drug reactions. The primary outcome measurements for efficacy assessment were changes from baseline in IPSS, IPSS-S, IPSS-V and IPSS-QoL after 6 months. The secondary outcome measurements for efficacy assessment were changes from baseline in AIS and MAQ.

\section{Statistical analysis}

All statistical analyses were carried out using SPSS 22.0 (IBM Corp., Armonk, NY, USA) software. We have analyzed whether parameters were normally distributed using Shapiro-Wilk test, $\alpha=0.05$. According to ShapiroWilk test age, PSA, BMI, IPSS, IPSS-S, IPSS-V, IIEF-5 are normally distributed, while TPV, Qmax, IPSS-QoL, AIS and MAQ are not. Normally distributed continuous variables are expressed as the mean \pm standard deviation, and non-normally distributed continuous variables are expressed as median [inter quartile range (IQR)]. The student's $t$-test and Mann-Whitney $\mathrm{U}$ test were used to compare the clinical indicators before and after medication, as well as the clinical data between the two groups. All tests were 2 -sided, and a $\mathrm{P}$ value of $<0.05$ was considered a statistically significant difference. We performed a perprotocol analysis (PP analysis). For continuous primary and secondary outcome variables, the Markov chain Monte Carlo multiple imputation method was used to impute missing data using 10 iterations.

\section{Results}

From September 2019 to July 2020, we recruited a total of 143 patients and excluded 23 patients, who did not meet the inclusion criteria $(n=11)$, refused to participate $(n=7)$, and excluded $(\mathrm{n}=5)$ for other reasons. A total of 105 patients completed the study, and the completion rate was $87.5 \%$. A total of 15 patients withdrew from the study, including 11 patients in the SM group [eight did not complete followup, one withdrew due to adverse drug reactions, and two underwent transurethral resection of the prostate (TURP)] and four in the Phellodendron Bawei tablets + SM group (three did not complete follow-up and one underwent TURP) (Figure 1). The adverse drug reactions that caused those in the SM group to withdraw were gastrointestinal in nature. Participants in both groups who withdrew to receive TURP did so due to acute urinary retention. There are no important harms or unintended effects in each group.

The baseline characteristics of the study population are shown in Table 1. There was no significant difference in age, BMI, PSA, PV, Qmax, IPSS score, IPSS-QOL score, AIS score, or IIEF-5 score between the two groups $(\mathrm{P}>0.05)$.

Table 2 shows the changes in clinical indicators and 


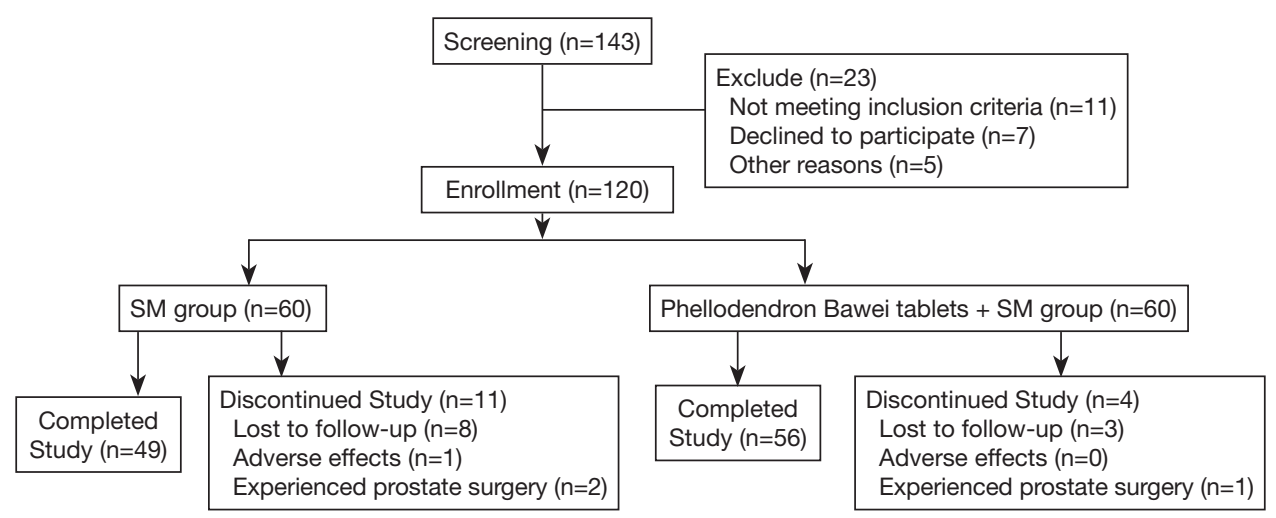

Figure 1 CONSORT diagram of the study.

Table 1 Comparison of demographic characteristics between SM and Phellodendron Bawei + SM groups

\begin{tabular}{lccc}
\hline Variables & SM & Phellodendron Bawei + SM & P value \\
\hline Patients & 49 & 56 & 0.292 \\
Age $(\mathrm{y})$ & $62.5 \pm 5.8$ & $25.5 \pm 2.8$ & 0.405 \\
BMI $\left(\mathrm{kg} / \mathrm{m}^{2}\right)$ & $26.0 \pm 3.1$ & $2.2 \pm 0.8$ & 0.677 \\
Total PSA $(\mathrm{ng} / \mathrm{mL})$ & $2.1 \pm 1.0$ & $45.5(21.8)$ & 0.108 \\
PV $(\mathrm{mL})$ & $38.5(11.9)$ & $9.4(3.6)$ & 0.625 \\
Qmax $(\mathrm{mL} / \mathrm{s})$ & $8.5(5.2)$ & $18.7 \pm 3.6$ & 0.185 \\
IPSS-total score & $17.8 \pm 3.4$ & $11.6 \pm 3.1$ & 0.309 \\
IPSS-V score & $11.0 \pm 3.0$ & $7.0 \pm 2.3$ & 0.576 \\
IPSS-S score & $6.7 \pm 2.2$ & $4.0(2.0)$ & 0.336 \\
IPSS-QOL score & $4.0(1.5)$ & $11.5(7.0)$ & 0.333 \\
AIS score & $11.0(5.5)$ & $15.7 \pm 4.1$ & 0.442 \\
IIEF-5 score & $15.1 \pm 4.4$ & & \\
\hline
\end{tabular}

SM, standard management; BMI, body mass index; PSA, prostate-specific antigen; PV, prostate volume; Qmax, maximum flow rate; IPSS, International Prostate Symptom Score; V, voiding subscore; S, storage subscore; QOL, quality of life; AIS, Athens Insomnia Scale; IIEF-5, International Index of Erectile Function-5.

relevant scores in the two groups after 6 months of treatment. PV, Qmax, IPSS scores, IPSS-V scores, IPSS-S scores, IPSS-QOL scores, and AIS scores of the two groups improved compared with the baseline values $(\mathrm{P}<0.05)$ after 6 months of treatment, but IIEF-5 scores of both groups decreased $(\mathrm{P}<0.05)$. This indicated that both drug regimens had good therapeutic effects on $\mathrm{BPH}$, but also a negative effect on sexual function.

Table 3 shows the changes in clinical indicators and relevant scores in control (SM) and experimental groups (Phellodendron Bawei tablets + SM) after 6 months of treatment. There was no significant difference in $\mathrm{PV}$ ( $\mathrm{P}=644)$, $\mathrm{Qmax}(\mathrm{P}=0.441)$, IPSS-total score $(\mathrm{P}=0.488)$, IPSS-V score $(\mathrm{P}=0.190)$, IPSS-QOL score $(\mathrm{P}=0.498)$, or IIEF-5 score $(\mathrm{P}=0.349)$ between the experimental group and control group, but IPSS-S $(\mathrm{P}=0.027)$ and AIS scores $(\mathrm{P}<0.001)$ were improved more significantly in the Phellodendron Bawei tablets + SM group, and the MAQ score in the Phellodendron Bawei tablets + SM group was lower $(\mathrm{P}=0.003)$. This indicates that compared with the control group, the experimental group had greater improvements in storage symptoms and sleep status, and 
Table 2 Within-group comparison of clinical parameters between SM and Phellodendron Bawei + SM groups

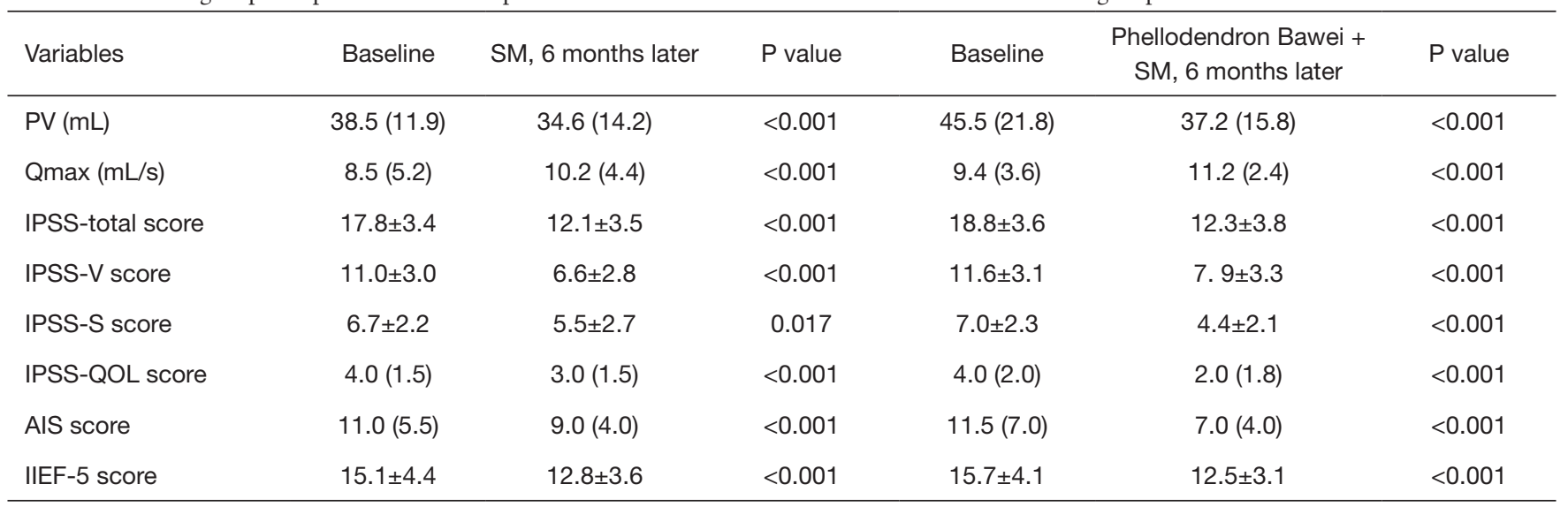

SM, standard management; PV, prostate volume; Qmax, maximum flow rate; IPSS, International Prostate Symptom Score; V, voiding subscore; S, storage subscore; QOL, quality of life; AIS, Athens Insomnia Scale; IIEF-5, International Index of Erectile Function-5.

Table 3 Comparison of clinical parameter changes between SM and Phellodendron Bawei tablets + SM groups

\begin{tabular}{lccc}
\hline Variables & SM & Phellodendron Bawei tablets + SM & P value \\
\hline Patients & 49 & 56 & 0.644 \\
PV $(\mathrm{mL})$ & $-6.7(12.1)$ & $-7.5(12.8)$ & 0.441 \\
Qmax (mL/s) & $2.0(1.5)$ & $2.4(2.9)$ & 0.488 \\
IPSS-total score & $-5.9 \pm 3.9$ & $-6.4 \pm 3.7$ & 0.190 \\
IPSS-V score & $-4.4 \pm 2.9$ & $-3.6 \pm 3.4$ & 0.027 \\
IPSS-S score & $-1.2 \pm 3.5$ & $-2.6 \pm 2.6$ & 0.498 \\
IPSS-QOL score & $-1.0(1.0)$ & $-1.0(1.8)$ & $<0.001$ \\
AIS score & $-2.0(3.0)$ & $-5.0(3.8)$ & 0.003 \\
MAQ score & $3.0(3.0)$ & $2.0(3.5)$ & 0.349 \\
IIEF-5 score & $-2.2 \pm 3.8$ & $-2.9 \pm 4.0$ & \\
\hline
\end{tabular}

SM, standard management; PV, prostate volume; Qmax, maximum flow rate; IPSS, International Prostate Symptom Score; V, voiding subscore; S, storage subscore; QOL, quality of life; AIS, Athens Insomnia Scale; MAQ, Medication Adherence Questionnaire; IIEF-5, International Index of Erectile Function-5.

the experimental group had higher medication compliance.

\section{Discussion}

Among older men, one of the most commonly seen conditions is BPH with predominant LUTs (16). The prevalence of clinical $\mathrm{BPH}$ in males over 50 years is $50-75 \%$, which increases with age, and the prevalence of clinical BPH in males over 70 years exceeds $80 \%$. Symptomatic and asymptomatic bacterial infections often occur in the male lower urinary and reproductive tract, which activate prostate-related lymphoid tissue in the prostate (17). Prostate-associated lymphoid tissue secretes a variety of cytokines, including interleukin-2 (IL-2), interleukin-8 (IL-8), interleukin-17 (IL-17), and interferon- $\gamma($ IFN- $\gamma)$. These cytokines, which are mostly detected at the early development stage, directly promote mitosis of the prostate, which leads to BPH $(18,19)$. The obstruction of the bladder outflow tract resulting from $\mathrm{BPH}$ and the subsequent residual urine infections lead to the reflux of infected urine into the prostate, further aggravating infections (20). 
The Phellodendron Bawei tablet is a proprietary Chinese medicine with anti-inflammatory and anti-infective effects. Its main components are Cortex Phellodendri, pine-soot ink, Fructus Gardeniae, Radix Glycyrrbiza, Flos Carthami, Fructus Piperis Longi, Pulvis Billis Bovis, and Resina Liquidambaris. Previous studies have confirmed that the berberine in Cortex Phellodendri regulates the energy metabolism of macrophages through the adenosine monophosphate-activated protein kinase/hypoxia-inducible factor-1 $\alpha$ (AMPK/HIF-1 $\alpha$ ) pathway (21) and also inhibits the interleukin-6/signal transducer and activator of

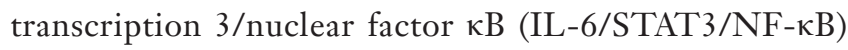
pathway (22). Further, dihydroberberine, a hydrogenated product of berberine, double regulates the NF- $\mathrm{\kappa B}$ and AMPK pathways (23). In addition, limonin in Cortex Phellodendri inhibits inducible nitric oxide synthase (iNOS) through nuclear factor $\mathrm{\kappa B}(\mathrm{NF}-\mathrm{\kappa B})$-mediated pathways (24). Inflammation can be alleviated through the regulation of any of the above-mentioned pathways.

The results of the present study showed that PV, Qmax, IPSS-total, IPSS-V, IPSS-S, and QOL scores of the patients in the two groups were significantly improved after drug treatment compared with the baseline values, but the improvement of IPSS-S scores in the SM + Phellodendron Bawei tablets group was greater than that in the SM group. This may be because the IPSS-S score is mainly related to prostatitis and Phellodendron Bawei tablets have a significant anti-inflammatory effect on prostatitis. This potential anti-inflammatory mechanism may be mediated by the downregulation of the mRNA expression of a group of inflammatory mediators, including TNF- $\alpha$, IL-1, IL-6, and COX-2 (25). Previous studies have reported that combination treatment with $\alpha 1$-receptor blockers and/ or $5 \alpha$-reductase inhibitors and other drugs results in more effective symptom relief and greater improvement in quality of life than the application of either treatment alone, with acceptable tolerance (26). This aligns with our research results. However, previous studies did not subdivide the improvement of symptoms into storage and voiding, nor did they discuss the impact on sleep quality $(27,28)$.

IPSS can be divided into the subgroups of IPSS-S and IPSS-V that correspond to symptoms of storage and voiding, respectively. The IPSS-S score-related symptoms (frequent micturition, urge incontinence, and nocturia) may be difficult and embarrassing for the patient, affecting their daily and social life. Nocturia, in particular, negatively affects sleep, increases fatigue, and adversely impacts the quality of life (29). Moreover, nocturia may also lead to a decline in the quality of life of patients' partners (30). Therefore, treatment of nocturia is also a focus of $\mathrm{BPH}$ treatment, but the effectiveness of the drugs, tamsulosin and finasteride, in treating nocturia is limited (31). Besides, when patients have a serious sleeping disorder, it may be necessary to use nonbenzodiazepine sedative-hypnotics, such as zolpidem (32). Although the incidence of adverse reactions associated with zolpidem is lower compared with that of benzodiazepine sedative-hypnotics, withdrawal reactions, hangover effect, headache, dizziness, nausea, drowsiness, and other adverse reactions may still occur (33).

We explored the effects of SM + Phellodendron Bawei tablets on the sleep quality and sexual function of patients with $\mathrm{BPH}$ for the first time. Although there was no significant difference in sexual function between the two groups after 6 months of medication, we found that the improvement of the AIS score in the SM + Phellodendron Bawei tablets group was greater than that in the SM group. Traditional Chinese medicine states that Flos Carthami nourishes the heart, calms nerves, promotes blood circulation, dredges collaterals, and can be used to treat insomnia due to qi deficiency in the blood; meanwhile, liquiritin, one of the main flavonoids in Radix Glycyrrbiza, strongly enhances the gamma-aminobutyric acid $\left(\mathrm{GABA}_{\mathrm{A}}\right.$ ) receptor expression, thus exerting a hypnotic effect (34). In addition, Phellodendron Bawei tablets improve LUTs during storage (including nocturia) and also indirectly improve the sleep quality of patients.

Interestingly, we also found that the medication compliance of patients was significantly improved after being administered Phellodendron Bawei tablets. Studies have shown that among Chinese Americans, traditional Chinese medicine prescriptions lead to higher compliance (35). This may be because patients with $\mathrm{BPH}$ are mostly middle-aged and older adult patients born in the 1960s, at that time traditional Chinese medicine was widely used in clinical practice with a high degree of recognition, leading to a higher acceptance rate of proprietary Chinese medicines. In addition, after administration, symptoms, sleep quality, and quality of life improved significantly in patients, which may also contribute to improving medication compliance.

However, our study also had some limitations. First, the research period was short, and the indicators were not observed dynamically at multiple time points. Second, this study was a single-center study with limited data. In the future, cooperation between multiple centers could be considered to expand the sample size. Finally, animal and 
cellular experiments were not conducted to explore the specific molecular mechanism of Phellodendron Bawei tablets in improving LUTs during storage and sleep quality.

In conclusion, $\alpha 1$-receptor blockers and $5 \alpha$-reductase inhibitors combined with Phellodendron Bawei tablets improve LUT symptoms during storage and sleep quality of patients, while increasing medication compliance compared to treatment with tamsulosin and finasteride. These results warrant further exploration to help combat the often debilitating effects of $\mathrm{BPH}$, particularly because of its growing prevalence.

\section{Acknowledgments}

Funding: This work was supported by the Natural Science Foundation of Hunan Province China (Grant No. 2020JJ5895). We would like to thank Editage (www.editage. cn) for English language editing.

\section{Footnote}

Reporting Checklist: The authors have completed the CONSORT reporting checklist. Available at https://dx.doi. org/10.21037/tau-21-588

Trial Protocol: Available at https://dx.doi.org/10.21037/tau21-588

Data Sharing Statement: Available at https://dx.doi. org/10.21037/tau-21-588

Conflicts of Interest: All authors have completed the ICMJE uniform disclosure form (available at https://dx.doi. org/10.21037/tau-21-588). The authors have no conflicts of interest to declare.

Ethical Statement: The authors are accountable for all aspects of the work in ensuring that questions related to the accuracy or integrity of any part of the work are appropriately investigated and resolved. The study was conducted in accordance with the Declaration of Helsinki (as revised in 2013). The study was approved by the Medical Ethics Committee of Xiangya Hospital of Central South University (approval No. 201703544) and informed consent was taken from all individual participants.

Open Access Statement: This is an Open Access article distributed in accordance with the Creative Commons
Attribution-NonCommercial-NoDerivs 4.0 International License (CC BY-NC-ND 4.0), which permits the noncommercial replication and distribution of the article with the strict proviso that no changes or edits are made and the original work is properly cited (including links to both the formal publication through the relevant DOI and the license). See: https://creativecommons.org/licenses/by-nc-nd/4.0/.

\section{References}

1. Shortridge E, Donatucci C, Donga P, et al. adherence and persistence patterns in medication use among men with lower urinary tract symptoms/benign prostatic hyperplasia. Am J Mens Health 2017;11:164-9.

2. Chen B, Cao D, Chen Z, et al.Estrogen regulates the proliferation and inflammatory expression of primary stromal cell in benign prostatic hyperplasia. Transl Androl Urol 2020;9:322-31.

3. Roehrborn CG, Barkin J, Tubaro A, et al. Influence of baseline variables on changes in International Prostate Symptom Score after combined therapy with dutasteride plus tamsulosin or either monotherapy in patients with benign prostatic hyperplasia and lower urinary tract symptoms: 4-year results of the CombAT study. BJU Int 2014;113:623-35.

4. Roehrborn CG, Siami P, Barkin J, et al. The effects of combination therapy with dutasteride and tamsulosin on clinical outcomes in men with symptomatic benign prostatic hyperplasia: 4-year results from the CombAT study. Eur Urol 2010;57:123-31.

5. Lokeshwar SD, Harper BT, Webb E, et al. Epidemiology and treatment modalities for the management of benign prostatic hyperplasia. Transl Androl Urol 2019;8:529-39.

6. Nygård LH, Talala K, Taari K, et al. The effect of nonsteroidal anti-inflammatory drugs on risk of benign prostatic hyperplasia. Prostate 2017;77:1029-35.

7. Busato WF, Almeida GL, Geraldo J, et al. Does PSA reduction after antibiotic therapy permits postpone prostate biopsy in asymptomatic men with PSA levels between 4 and $10 \mathrm{ng} / \mathrm{mL}$ ? Int Braz J Urol 2015;41:329-36.

8. Vinarov AZ, Spivak LG, Platonova DV, et al. 15 years' survey of safety and efficacy of Serenoa repens extract in benign prostatic hyperplasia patients with risk of progression. Urologia 2019;86:17-22.

9. Alcaraz A, Carballido-Rodriguez J, Unda-Urzaiz M, et al. Quality of life in patients with lower urinary tract symptoms associated with BPH: change over time in real- 
life practice according to treatment--the QUALIPROST study. Int Urol Nephrol 2016;48:645-56.

10. Sun Y, Lenon GB, Yang A. Phellodendri Cortex: a phytochemical, pharmacological, and pharmacokinetic review. Evid Based Complement Alternat Med 2019;2019:7621929.

11. Singh A, Zhao K. Treatment of insomnia with traditional Chinese herbal medicine. Int Rev Neurobiol 2017;135:97-115.

12. Liu H, Zhao CY, Zhang W, et al. Study on medication laws of Tibetan medicine in treatment of plateau disease based on data mining technology. Zhongguo Zhong Yao Za Zhi 2018;43:1726-31.

13. van Kollenburg RA, de Bruin DM, Wijkstra H. Validation of the electronic version of the international index of erectile function (IIEF-5 and IIEF-15): A crossover study. J Med Internet Res 2019;21:e13490.

14. Lin CY, Cheng A, Nejati B, et al. A thorough psychometric comparison between Athens Insomnia Scale and Insomnia Severity Index among patients with advanced cancer. J Sleep Res 2020;29:e12891.

15. Zabkowski T, Saracyn M. Drug adherence and drugrelated problems in pharmacotherapy for lower urinary tract symptoms related to benign prostatic hyperplasia. J Physiol Pharmacol 2018. doi: 10.26402/jpp.2018.4.14.

16. Wang B, Zhang S, Sun C, et al.Comparison between a transurethral prostate split and transurethral prostate resection for benign prostatic hyperplasia treatment in a small prostate volume: a prospective controlled study. Ann Transl Med 2020;8:1016.

17. Chughtai B, Forde JC, Thomas DD, et al. Benign prostatic hyperplasia. Nat Rev Dis Primers 2016;2:16031.

18. Wang L, Zoetemelk M, Chitteti BR, et al. Expansion of prostate epithelial progenitor cells after inflammation of the mouse prostate. Am J Physiol Renal Physiol 2015;308:F1421-30.

19. Aaron L, Franco OE, Hayward SW. Review of prostate anatomy and embryology and the etiology of benign prostatic hyperplasia. Urol Clin North Am 2016;43:279-88.

20. Ledda A, Belcaro G, Dugall M, et al. Supplementation with high titer cranberry extract (Anthocran(R)) for the prevention of recurrent urinary tract infections in elderly men suffering from moderate prostatic hyperplasia: a pilot study. Eur Rev Med Pharmacol Sci 2016;20:5205-9.

21. Yu Y, Cai W, Zhou J, et al. Anti-arthritis effect of berberine associated with regulating energy metabolism of macrophages through AMPK/ HIF-1 $\alpha$ pathway. Int Immunopharmacol 2020;87:106830.
22. Zhu L, Gu P, Shen H. Protective effects of berberine hydrochloride on DSS-induced ulcerative colitis in rats. Int Immunopharmacol 2019;68:242-51.

23. Tan L, Wang Y, Ai G, et al. Dihydroberberine, a hydrogenated derivative of berberine firstly identified in Phellodendri Chinese Cortex, exerts antiinflammatory effect via dual modulation of NF-kappaB and MAPK signaling pathways. Int Immunopharmacol 2019;75:105802.

24. Fujii A, Okuyama T, Wakame K, et al. Identification of anti-inflammatory constituents in Phellodendri Cortex and Coptidis Rhizoma by monitoring the suppression of nitric oxide production. J Nat Med 2017;71:745-56.

25. Xian YF, Mao QQ, Ip SP, et al. Comparison on the antiinflammatory effect of Cortex Phellodendri Chinensis and Cortex Phellodendri Amurensis in 12-O-tetradecanoylphorbol-13-acetate-induced ear edema in mice. J Ethnopharmacol 2011;137:1425-30.

26. Xu XF, Liu GX, Zhu C, et al. alpha1-Blockers and 5alphaReductase Inhibitors Are the Most Recommended Drugs in Treating Benign Prostatic Hyperplasia: An EvidenceBased Evaluation of Clinical Practice Guidelines. Front Pharmacol 2020;11:311.

27. Alcaraz A, Rodríguez-Antolín A, Carballido-Rodríguez J, et al. Clinical benefit of tamsulosin and the hexanic extract of serenoa repens, in combination or as monotherapy, in patients with moderate/severe LUTS-BPH: A subset analysis of the QUALIPROST study. J Clin Med 2020;9:2909.

28. Sokhal AK, Sankhwar S, Goel A, et al. A prospective study to evaluate sexual dysfunction and enlargement of seminal vesicles in sexually active men treated for benign prostatic hyperplasia by alpha-blockers. Urology 2018;118:92-97.

29. Bliwise DL, Rosen RC, Baum N. Impact of nocturia on sleep and quality of life: a brief, selected review for the International Consultation on Incontinence Research Society (ICI-RS) nocturia think tank. Neurourol Urodyn 2014;33:S15-18.

30. Marklund H, Spangberg A, Edell-Gustafsson U. Sleep and partner-specific quality of life in partners of men with lower urinary tract symptoms compared with partners of men from the general population. Scand J Urol 2015;49:321-28.

31. Oelke M, Fangmeyer B, Zinke J, et al. Nocturia in men with benign prostatic hyperplasia. Aktuelle Urol 2018;49:319-27.

32. Miwa K, Nishino Y, Kikuchi M, et al. Efficacy of combination therapy with tamsulosin and zolpidem on 
nocturia in patients with benign prostatic hyperplasia.

Cent European J Urol 2011;64:232-5.

33. Monti JM, Spence DW, Buttoo K, et al. Zolpidem's use for insomnia. Asian J Psychiatr 2017;25:79-90.

34. Hoffmann KM, Beltran L, Ziemba PM, et al. Potentiating effect of glabridin from Glycyrrhiza glabra on GABAA

Cite this article as: Gong YC, Chen X, Song QT, Gan Y, Zhang B, Li BS, Chen Z, He Y. A randomized placebocontrolled study: Phellodendron Bawei tablets combined with standard management can improve storage symptoms, sleep quality, and medication compliance in patients with benign prostatic hyperplasia compared to placebo with standard management. Transl Androl Urol 2021;10(8):3423-3431. doi: $10.21037 /$ tau-21-588 receptors. Biochem Biophys Rep 2016;6:197-202.

35. Sun K, Szymonifka J, Tian H, et al. Association of traditional Chinese medicine use with adherence to prescribed western rheumatic medications among Chinese American patients: A cross-sectional survey. Arthritis Care Res (Hoboken) 2020;72:1474-80. 